\title{
Radiation Reduction of Carbon Dioxide: A New Chemical Industry?
}

\author{
Peter M. Livingston* \\ 1321Via Zumaya, Palos Verdes Estates, California
}

A new carbon dioxide reduction method using the products of hard radiation is proposed. The radiation is supplied by spent fuel rod assemblies deep in 'cooling ponds' located next to nuclear power plants. This is a unique untapped energy source with no carbon footprint and available in very plentiful supply. The actual radiochemistry occurs in specially designed reaction vessels filled with water located surrounding a spent fuel rod assembly. Carbon dioxide and reducer gases are bubbled through the reaction vessel water where they are subject to the energetic electron flux created by gamma ray deposit. A modification of the chemical potentials dependent on the energetic electron density in water created by a known gamma ray spectrum among other parameters, determine the carbon dioxide reaction rate. A typical filled-to-capacity cooling pond houses a thousand fuel rod assemblies. The simplest reductant is hydrogen gas and the desired product is carbon monoxide. Calculations reveal that such a pond could deliver a little over a half megatonne of carbon monoxide a year. When dry-cask containers for spent fuel rod assemblies are permitted transport to long term storage, it will also be possible to transport them to new deep pond facilities not located in conjunction with nuclear facilities. Now there is no limitation on size scaling and a new competitive radiochemical industry is created.

\section{Introduction}

Carbon dioxide, like all molecules, fragments when struck by gamma rays, Compton electrons and their progeny. But, like water itself, the fragments very rapidly reassemble in most all cases. This reassembly process is interrupted in carbon dioxide by adding to it a hydrogen donor molecule that successfully competes with the $\mathrm{CO}$ fragment for the released oxygen. This molecule can be elementary hydrogen gas, or other simple molecules described in the text below. It is the purpose of this article to show not only the basis for this claim, but to propose a workable system to exploit radiation carbon dioxide reduction that can be scaled to industrial size.

It is common knowledge that, over the next few decades, capturing carbon dioxide from high concentrated streams such as flue gases from power and cement plants, and from the atmosphere itself could become a viable factor in controlling global warming. No one technology is a magic bullet, and it will undoubtably take every action from direct chemical to biomass capture to make a dent in present atmospheric concentrations. However, it is imperative that current fossil carbon remains in the ground, be it oil, gas or coal, to be replaced by renewable energy sources; fuels derived from repurposed carbon; and importantly feedstocks for the chemical industry that supports much of our present civilization.

Present day management of carbon dioxide (if it is managed at all), links the capture technology to long term storage in which the captured $\mathrm{CO}_{2}$ is forced underground in deep wells. There it is hoped, the injected gas will bind to the substrate rock to sequester it. Carbon dioxide deep well storage is a new technology with only several years-experience. High pressure liquid injection into underground wells as part of tertiary oil recovery has resulted in quakes and gas leaks into 
the water-bearing aquifer layer above the storage site. This could also happen to $\mathrm{CO}_{2}$ well storage.

Over the past decade or more, many articles have been published on reducing carbon dioxide with the aid of catalysts $1,2,3,4$. While these methods undoubtably work, they also require some external energy supply and are subject to catalyst poisoning if the captured gas is contaminated with sulfur or organometallic compounds. This is a problem solved by suitably cleansing the gas, but at a cost. Further, there is a warning in the literature about employing rare metals ${ }^{5}$.

The energy supply requirement raises a caution flag: if the latter is generated by fossil fuels, then a carbon 'footprint' is created in the atmosphere which reduces the beneficial carbon sequestration effort.

Nonetheless, forward leaning companies are looking to profit from carbon dioxide capture according to a recent article in State of the Planet, Earth Institute, Columbia University ${ }^{6}$. In the near term, $\mathrm{CO}_{2}$ incorporation into concrete is a commercially viable option. But the article also noted that (present day) fossil fuel manufacture of organic chemicals that make up solvents, synthetic rubber, plastics and more, is responsible for about 2 gigatonnes of $\mathrm{CO}_{2}$ emissions per year. The article goes on to explain that these materials can also be made from carbon dioxide, but in all cases the manufacturing process requires the addition of heat energy.

We propose another approach to repurposing carbon in carbon dioxide that uses an untapped, non-polluting energy source and requires no catalysis: employ gamma ray radiation as the prime energy source. There is a plentiful supply available in nuclear power plant storage ponds: highly radioactive nuclear fuel rod assemblies no longer capable of slow-neutron fission. They are 'cooled' there for a year or more before they can be safely shipped in cement caissons to some, yet undetermined storage location.

The radiochemistry basis for carbon dioxide reduction.

Experiments exposing carbon dioxide to radiation date back to the advent of the x-ray tube at the turn of the twentieth century. Further information on the impact of hard radiation on carbon dioxide emerged when it was chosen as a heat transfer agent in the first British graphite moderated/natural uranium pile, $\mathrm{MAGNOX}^{7}$. It was discovered that carbon dioxide, like water, is stable in an intense hard radiation field because the dissociated molecules rapidly reconstitute themselves.

In order to sort out the $\mathrm{CO}_{2}$ fragments created by hard radiation, physical chemists ${ }^{8,9,10,11}$ inhibited the back reaction by employing a variety of hydrogen donors. They found that $\mathrm{CO}_{2}$ in an aqueous medium decomposed by $\mathrm{MeV}$ photons from a ${ }^{60} \mathrm{Co}$ source yielded these fragments:

$$
7.53 \mathrm{CO}_{2} \rightarrow 0.007 \mathrm{C}^{+}+0.51 \mathrm{CO}^{+}+0.21 \mathrm{O}^{+}+2.24 \mathrm{CO}_{2}^{+}+4.71 \mathrm{CO}+5.16 \mathrm{O}+3.03 e^{-}
$$

Firstly, note that $\mathrm{CO}$ is relatively immune from further decomposition as indicated by the small $\mathrm{C}^{+}$relative contribution. That also implies that production of solid carbon suboxide will be negligible. Secondly, note the large concentration of atomic oxygen that will be the target of various hydrogen donors. 
The reaction above has been numerically modelled in a 1977 article published in Journal of Physical Chemistry ${ }^{12}$.

Radiochemists quantify the number of a specific molecular fragments per $100 \mathrm{eV}$ of incident hard radiation by specifying a parameter, G. A $G$ value of 4 for example, means that for every 1 $\mathrm{MeV}$ photon that is absorbed $4 \times 10^{4}$ fragments of a specific type are created.

$\mathrm{G}$ values were measured with various oxygen scavenger molecules, such isopropanol, and acetone yielding $\mathrm{G}(\mathrm{CO})$ 's $\sim 4$ through 8 depending on the carbon dioxide partial pressure. The scavenger substances were present in a few per cent concentration. Additionally, the aqueous solution provides intimate contact between the fast electrons produced in the gamma ray Compton scattering decay in water: Each Compton event creates a gamma ray of lesser energy plus a fast electron. Numerical experiments indicate $3 \sim 4$ thousand daughter gamma photons are created for every primary gamma deposit in the reactor water. Each of these inelastically scattering from an electron emit an energetic electron at an energy and momentum determined by the gamma ray scattering angle.

Two earlier papers ${ }^{13,14}$ reported the reduction of carbon dioxide in both pure gas form and aqueous solutions by ionizing radiation created by high energy electron pulses. A G(CO) value of $7.8 \pm 0.3$ in one case was obtained showing the effect of fast-electron bond breakage- the principal mechanism by which carbon dioxide is broken apart. It closely represents what happens in our bubble reactor concept described below.

Maximum radiation coupling to the gases

One practical problem to be solved is how best to couple the radiation to a gas, given its low density relative to a liquid or solid. Norihiko Fujita added iron powder to an aqueous solution of carbon dioxide in water both to increase gamma capture and supply an oxygen scavenger molecule $^{15}$. Radiation induced corrosion of the iron led to a supply of hydrogen that scavenged the oxygen from a decomposed carbon dioxide molecule. Our approach to this problem is to bubble the reactant gases through water (isolated from the cooling pond water) in a specially designed reactor vessel described below. Now the gases are completely immersed in the fast electron flux created by gamma deposit in the vessel's water.

Radiation safety is an issue

Radiation safety is a paramount concern and is satisfied by conducting the carbon dioxide reduction deep within the cooling pond itself. The fuel rod assemblies are not moved by this process; therefore, a fully instrumented cooling pond has no additional radiation concerns and is as safe as the original pond itself.

The Radiation Source

We assume our radiation source is a PWR (Pressurized Water Reactor) fuel rod assembly consisting of 271 fuel pins bundled in a $22 \mathrm{~cm} \times 22 \mathrm{~cm} \times 400 \mathrm{~cm}$ array. The burnup is 45MDay/tU ( $\mathrm{tU}$ : tonnes uranium). A typical assembly has been sitting in the cooling pond for six months or so, ridding it of the short-lived isotopes. Table 1 show a list of the remaining significant isotopes and their activity. 
Most of the isotopes emit beta particles leaving the end-product in an excited state. This effect is accounted for (branching ratios) in determining the gamma emissions as shown in columns 4 and 5 . The last column represents gamma emissions normalized to that of ${ }^{137} \mathrm{Cs}$.

\begin{tabular}{|c|c|c|c|c|c|}
\hline \multicolumn{6}{|c|}{ Table 1. Radiation Source } \\
\hline Nuclides & Activity (Bq) Half-life & Mode, Br ratio\% & gamma/sec & $(\mathrm{MeV})$ & @ (6mo) \\
\hline Zr 95 & $1.91 \mathrm{E}+1964 \mathrm{day}$ & double beta (49\%) & $9.36 E+18$ & 0.724 & 0.212 \\
\hline Ru 106 & $9.60 E+18374$ days & beta (21\%) & $2.02 E+18$ & 0.512 & 0.485 \\
\hline Cs 134 & $3.80 E+182.07 y r$ & gamma (91\%) & $3.76 E+18$ & 0.796 & 1.16 \\
\hline Cs 137 & $3.00 \mathrm{E}+1830.2 \mathrm{yr}$ & gamma, beta (85\%) & $2.55 E+18$ & 0.667 & 1 \\
\hline Ce 143 & $1.72 E+19285$ days & beta, $(46 \%)$ & $7.91 E+18$ & 0.293 & 1.63 \\
\hline Eu 154 & $1.42 E+1716 y r s$ & gamma (40\%) & $5.68 E+16$ & 0.123 & 0.0223 \\
\hline
\end{tabular}

The maximum absorption of gamma rays in water is at the peak of the product of absorption and transmission for that photon energy. From the table 2 below, the average e-folding distance is $8.046 \mathrm{~cm}$; the peak is easily computed to be $8.046 / \sqrt{2}=5.69 \mathrm{~cm}$. On the other hand, there is quite a spread in gamma energies with a similar spread in energetic electron spectrum.

The Bubble Reactor

Our coupling problem resolution is the bubble reactor. Let the chemical reactor vessel be filled with water-isolated from the cooling pond water-and let the carbon dioxide, reducer gas-mixture bubble up through vessel in the presence of a radiation field. The vessel diameter is limited by other fuel rod assemblies nearby, but its height is determined by the required exposure of the fine bubbles to the fast electron flux. For a planned hole diameter of a fraction of a millimeter, the bubble size, and hence its rise time is simply determined from a buoyancy calculation in the laminar flow regime.

The bubble reactor is illustrated and described in Figure 2, shown here. It can be used for either products that are immiscible with water ( $\mathrm{CO}$ among others) or miscible with water (alcohols, aldehydes). The vessel height is set by the expected reaction such that by the time the tiny bubbles reach the top of the reactor vessel, the conversion from reactants to products is virtually complete. If some or all reaction products are water-soluble then the reactor water must be recycled through some extraction process such

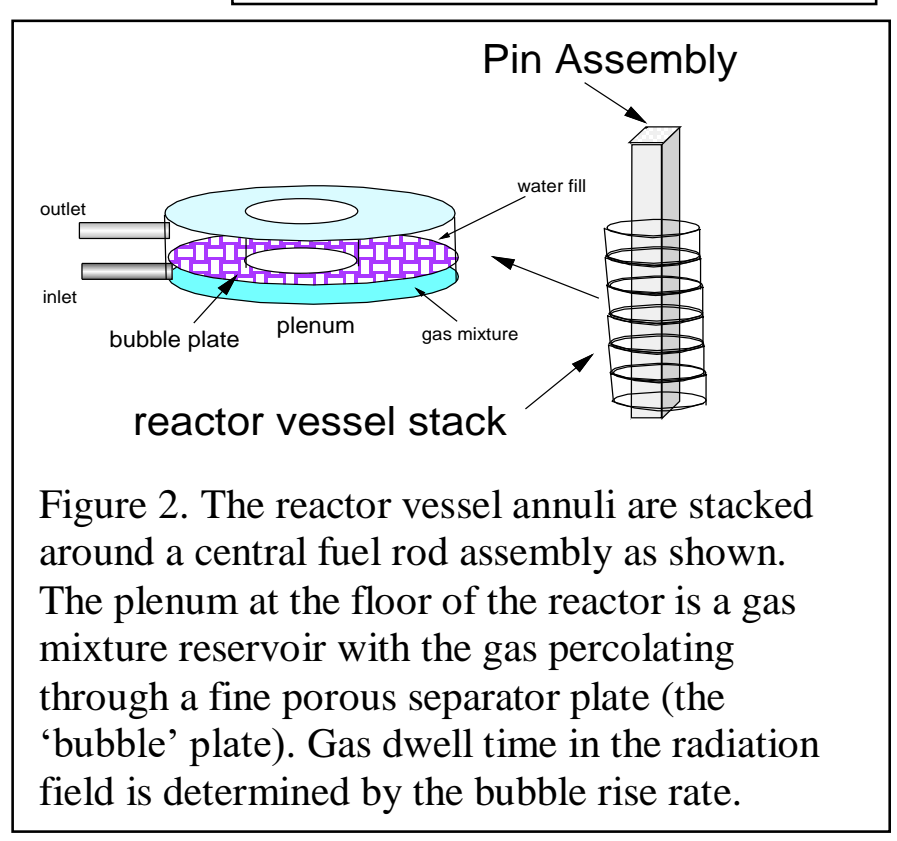

Figure 1. A typical power plant cooling pond.

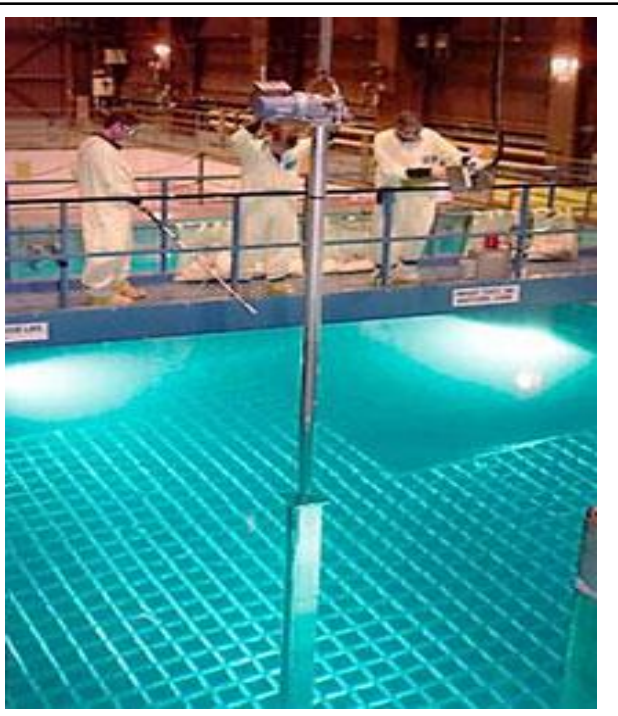


as benzene extraction or multiplate distillation. A soluble product also is a phase change that drives the reaction to completion.

The advantage of a bubble chemical reactor becomes apparent: (a) there is no physical barrier between the reactants and the gamma ray-generated fast electron flux in water; and (b), the latter is well-understood. Therefore, it is safe to assume that the bubble gases 'see' the same electron flux as present in the annulus water.

Figure 3 illustrates the 'packing' of the annuli by looking down on the array. As shown, each annulus receives radiation equivalent to three nearest neighbors, plus a factor of one-half accounting for

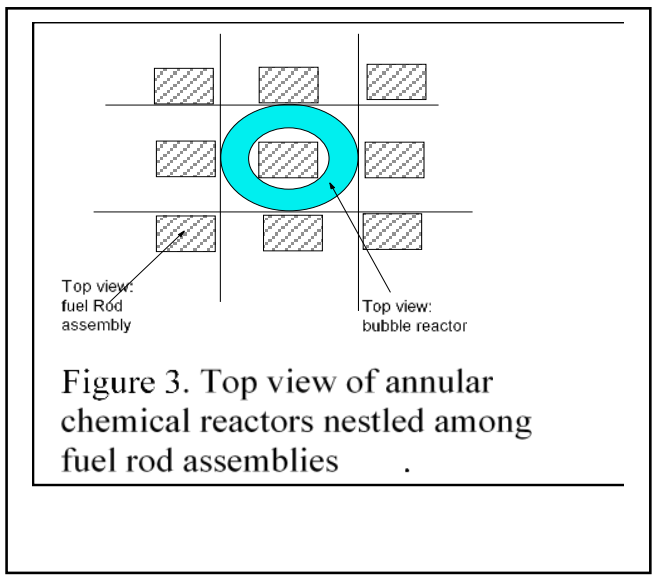
more distant fuel rod assemblies.

\section{Analysis}

The objective is to estimate the production rate of the radiation-induced products using the apparatus described above. Therefore, both a radiation-induced modification of the equilibrium constant for a given reaction, and an estimate of the fast electron flux in the reaction vessel water and are required.

The chemical potential for a chemical species is a useful construct in chemistry and is defined as the derivative of the Gibb's energy (aka Gibbs Free energy) with respect to the number of moles of that species. It is an intensive thermodynamic variable with the units of energy per mole.

This definition is unchanged when the chemical reactants are subject to gamma irradiation, although reference state does.

Consider a hypothetical gas phase homogeneous reaction

$$
n_{1} a_{1}+\ldots+n_{m} b_{m} \rightarrow n_{m+1} c_{m+1}+\ldots+n_{m+1} d_{m+1}
$$

Here $\mathrm{a}, \mathrm{b}, \mathrm{c}$, and $\mathrm{d}$ represent the chemical constituents.

Further let $n_{i}, I=1 \ldots q$ denote the moles of each constituent and $\mu_{i}, i=1 \ldots q$, the chemical potentials. Further let $\beta$ represent a common mole proportionality factor such that

$$
\begin{aligned}
& d n_{i}=-v_{i} d \beta \quad \text { if } i=1 \ldots m \\
& d n_{i}=v_{i} d \beta \quad \text { if } i=m+1 \ldots q
\end{aligned}
$$

Here the $v_{i}$ are small whole numbers denoting the molar ratio of the various chemicals taking part in the reaction and $\beta$ is an arbitrary common mole factor. The sign change between reactants and products is a convention for exothermic (energetically favored) reactions.

From the definition of the chemical potential $\mu$ as the partial derivative of the Gibbs energy with respect to the mole concentrations, the total differential $\mathrm{G}$ is now 


$$
d G=\sum_{i} \mu_{i} d n_{i}=\sum_{i} \mu_{i} v_{i} d \beta
$$

The equilibrium state is defined by

$$
\begin{aligned}
& \frac{\partial G}{\partial \beta}=0 \\
& \therefore \sum_{i} v_{i} \mu_{i}=0
\end{aligned}
$$

The second equation automatically subtracts the sum of the reactant chemical potentials from the product sum based on the indexing shown in eq. 3 .

The chemical potential for a pure gas constituent is defined as the reference chemical potential minus an entropy term:

$$
\mu_{i}(T, P)=\mu_{i}^{0}(T)-R T \ln \frac{p_{i}}{P_{0}}
$$

Here $\mathrm{p}_{\mathrm{i}}$ is the partial pressure of the $\mathrm{i}$-th constituent, and $\mathrm{P}_{0}$ is the reference pressure, usually taken as 1 atmosphere.

The effects of radiation on the gas mixture is accounted for by modifying the standard chemical potential.

$$
\mu_{i}^{0}(T, \tau) \equiv \mu_{i}^{0}(T)\left[1-\alpha_{i} f \tau\right]
$$

The three new factors are: a reduced atomic number a (relative electron density of the species); the fraction of molecules fragmented per second in the vessel $\mathrm{f}$, and the dwell time $\tau$ that a parcel of gas has spent in the radiation field. Here we make a tacit assumption: the scattering cross sections of the molecular electrons are all the same, independent of the fast-electron's energy. In fact, that is not an accurate statement, for the electron-electron scattering cross sections do depend on the collisional energy as well. However, we will accommodate this inaccuracy by defining the electron impact molecular fragmentation by the radiation chemist's $G$ value defined as the number of radiation-induced reactions per $100 \mathrm{eV}$ of electron impact energy-thus bypassing a difficult bookkeeping effort.

The reduced atomic number factor is the fraction of total electrons for constituent $i$ on either the reactant or product side (these are equal by mass conservation). It accounts for the fact that if some molecule has been fragmented, then a times that number is the conditional probability that it was the i-th type.

The factor $\mathrm{f}$ is the fraction of all molecules in the vessel that are fragmented per second. It is computed by averaging the probability of gamma capture in the surrounding reaction vessel water times the G-factor times the radiant spectrum times the vessel wall attenuation factor (if 
any) over all gamma energies. Lastly the dwell time $\tau$ (seconds) is easily converted to a flow rate so that ultimately, the model computes flow speeds needed for maximum product output.

The definition of equilibrium, (eq. 5); the chemical potential, (eq. 6); and its modification (eq. 7); combine to express the relation between the equilibrium constant, $\mathrm{K}$, and the molar Gibbs energy, $\Delta \mathrm{G}^{0}(\mathrm{P}, \mathrm{T}, \tau)$.

$$
\begin{aligned}
& K \equiv\left[\frac{\prod_{j} p_{j}^{v_{j}}}{\sum_{j^{j}} v_{j}}\right] \\
& 0=\sum_{j} v_{j} \mu_{j}^{0}(T, P, \tau)+R T \ln K \\
& \Delta G^{0}=\sum_{j} v_{j} \mu_{j}^{0}(T, P, \tau) \\
& K=e^{-\frac{\Delta G^{0}}{R T}}
\end{aligned}
$$

Suppose the reactants range from $\mathrm{j}=1$ to $\mathrm{m}$ and the products range from $\mathrm{j}=\mathrm{m}+1$ to $\mathrm{q}$. Assume that all the gas partial pressures are determined by the Perfect Gas Equation of State. Let $\chi_{j}$ be the mole fraction of the $\mathrm{j}$-th constituent. Then the equilibrium constant becomes:

$$
K \equiv\left[\frac{\prod_{j} p_{j}^{v_{j}}}{P_{0}^{\sum_{j} v_{j}}}\right]=\frac{\prod_{j=m+1}^{q} \chi_{j}^{v_{j}}}{\prod_{j=1}^{p} \chi_{j}^{v_{j}}} P_{0}^{q} \sum_{j=1+m}^{q} v_{j}-\sum_{j=1}^{m} v_{j}
$$

The Gibbs energy $\Delta \mathrm{G}^{0}$ from (8) now has an additional factor

$$
\begin{aligned}
\Delta G^{0} & =\sum_{j=m+1}^{q} v_{j} \mu_{j}^{0}-\sum_{j=1}^{m} v_{j} \mu_{j}^{0}-\left(\sum_{j=m+1}^{q} v_{j} \mu_{j}^{0} \alpha_{j}-\sum_{j=1}^{m} v_{j} \mu_{j}^{0} \alpha_{j}\right) f \tau \\
& =\Delta \tilde{G}^{0}-\Delta g f \tau
\end{aligned}
$$

The second line shows that the reaction Gibbs energy per mole is reduced by energy derived from the high energy electron bombardment shown as the second term.

Note that the factor $\exp (\Delta \mathrm{gf} \tau)$ forms a prefactor multiplying the radiation free equilibrium constant.

$$
K(T, \tau)=e^{\frac{\Delta g f \tau}{R T}} K^{0}(T)
$$

Provided $\Delta \mathrm{g}$ is positive (in most cases examined it is, but there are exceptions), then the equilibrium constant $\mathrm{K}$ favors the products over the reactants, forcing the reaction nearer to completion. This radiation 'forcing' is enough to convert many endothermic reactions into 
exothermic ones, making otherwise thermodynamically unfavorable products available. An example of this is the simple reverse water gas shift reaction in which equal molar amounts of hydrogen and carbon dioxide react to produce equal molar amounts of carbon monoxide and water driven by Compton electrons and their daughters.

We make the transition from endothermic to exothermic reaction clearer by tying the dwell time $\tau$ to the reaction extent $\xi$ that varies between 0 (no products) and 1 (no reactants). The latter is a bookkeeping device that tracks appropriate amounts of product and reactant concentrations.

Apart from a common factor the concentrations of the constituents are determined by the extent of reaction.

$$
\begin{array}{ll}
n_{j}(\xi)=v_{j}(1-\xi) & j=1 \ldots m \\
n_{j}(\xi)=v_{j} \xi & j=m+1 \ldots q \\
N(\xi)=\sum_{j=1}^{m} v_{j}(1-\xi)+\sum_{j=m+1}^{q} v_{j} \xi \\
\chi_{j}(\xi)=\frac{n_{j}(\xi)}{N(\xi)} \quad j=1 \ldots q
\end{array}
$$

The first two lines define the concentrations in terms of the reaction extent; the third line is proportional to the total number of moles in the reaction and the last line defines the mole fractions.

In general, the explicit solution for $\xi$ in terms of $\tau$ is the root of a polynomial expression shown in eq. 13 below. It requires a numerical solution except in very simple cases. However, that is no barrier because root-finding algorithms are commonplace.

$$
\prod_{j=m+1}^{q} \chi_{j}^{v_{j}}(\xi(\tau))-\prod_{j=1}^{m} \chi_{j}^{v_{j}}(\xi(\tau)) P^{\sum_{j=1}^{m} v_{j}-\sum_{j=1+m}^{q} v_{j}} K_{0}(T) e^{\frac{\Delta g f \tau}{R T}}=0
$$

Reaction rate in the radiation field

Here we determine the radiation field parameters; $f$ (fraction of molecules fragmented per second), $\tau$ (dwell time in the radiation field), and $\alpha$ (relative electron fraction for a given species). The latter is a rough measure of the probability that this molecule will be dismembered by a fast electron. We assume our radiation source is a PWR fuel rod assembly shown in Table 1.

Most of the isotopes emit beta particles leaving the end-product in an excited state. This effect is accounted for (branching ratios) in determining the gamma emissions as shown in columns 4 and 5. The last column represents gamma emissions normalized to that of ${ }^{137} \mathrm{Cs}$. 
The peak absorption of gamma rays in water is at the peak of the product of absorption and transmission for that photon energy. From Table 2, the average e-folding distance is $8.046 \mathrm{~cm}$;

\begin{tabular}{|l|r|r|r|r|r|r|r|}
\hline & Water, absorber & & & & & \\
\hline Isotope & E gamma & $\mathrm{cm}^{\wedge}$ 2/gm & e-folding, cm & rel. flux & weighted e-folding & gamma/sec & weighted \\
\hline Eu 154 & 0.123 & 0.565 & 1.77 & 0.0233 & 0.041241 & $5.68 \mathrm{E}+16$ & $1.32 \mathrm{E}+15$ \\
\hline Ce 143 & 0.293 & 0.119 & 8.4034 & 1.63 & 13.697542 & $7.91 \mathrm{E}+18$ & $1.29 \mathrm{E}+19$ \\
\hline Ru 106 & 0.512 & 0.09569 & 10.45 & 0.485 & 5.06825 & $2.02 \mathrm{E}+18$ & $9.80 \mathrm{E}+17$ \\
\hline Cs 137 & 0.667 & 0.08533 & 12.16 & 1 & 12.16 & $2.55 \mathrm{E}+18$ & $2.55 \mathrm{E}+18$ \\
\hline Zr 95 & 0.724 & 0.08224 & 12.16 & 0.212 & 2.57792 & $9.36 \mathrm{E}+18$ & $1.98 \mathrm{E}+18$ \\
\hline Cs 134 & 0.796 & 0.07875 & 12.7 & 1.16 & 14.732 & $3.76 \mathrm{E}+18$ & $4.36 \mathrm{E}+18$ \\
\hline & & & & Average & 8.046158833 & Average & $3.80 \mathrm{E}+18$ \\
\hline
\end{tabular}

Table 2. E-folding lengths for gammas in water generated by various isotopes in the fuel rod $\because$

the peak is easily computed to be $8.046 / \sqrt{ } 2=5.69 \mathrm{~cm}$. On the other hand, there is quite a spread in gamma energies with a similar spread in energetic electron spectrum.

The G-value for water determines the fast electron flux through the bubbles. The Canadian reference (16) establishes the decomposition/recomposition of water as 0.43 micromoles/joule that converts to a $\mathrm{G}$ value of 4.9 .

Equation 14 is a reduction of Table 1 in matrix form

$$
H_{1}=\left[\begin{array}{ccc}
0.212 & 0.724 & 9.36 \cdot 10^{18} \\
0.485 & 0.512 & 2.02 \cdot 10^{18} \\
1.16 & 0.796 & 3.76 \cdot 10^{18} \\
1.000 & 0.667 & 2.55 \cdot 10^{18} \\
1.630 & 0.293 & 7.91 \cdot 10^{18} \\
0.223 & 0.123 & 5.68 \cdot 10^{16}
\end{array}\right]
$$

$$
\begin{aligned}
& G_{\mathrm{H}_{2} \mathrm{O}}=4.9 \\
& V o l=50.3 \mathrm{~cm}^{3} \\
& t=323{ }^{0} \mathrm{~K} \\
& T_{\gamma}=\frac{\left(3.5 \cdot 10 G_{H_{2} O} \cdot 0.9\right) \sum_{j=0}^{5} H_{1_{j, 0}} H_{1_{j, 1}} H_{1_{j, 2}}}{V o l}=3.34363 \cdot 10^{19} \mathrm{~cm}^{-3} \mathrm{sec}^{-1} \\
& N=\frac{2 N_{a v}}{R_{a} t 1000}=4.54 \cdot 10^{19} \mathrm{~cm}^{-3} \\
& f=\frac{T_{\gamma}}{N}=\frac{3.34363 \cdot 10^{19}}{4.54 \cdot 10^{19}}=0.7365 \mathrm{sec}^{-1}
\end{aligned}
$$

The expressions shown in (eq. 15) above summarize the computation. The G-value for water is taken from the Canadian work ${ }^{16}$ cited above. "Vol" is that associated with a single "pancake 
reactor' illustrated in Fig. 1 with a height determined by the choice of bubble size, described below. The factor 3.5 follows by accounting for radiation from the central assembly and those surrounding it. The factor of 10 is the ratio of the number of $100 \mathrm{eV}$ intervals per $1 \mathrm{MeV}$ divided by the number of cubic centimeters per liter: e.g. $10^{4} / 10^{3}$. Gamma capture efficiency is denoted by 0.9 . Further, $\mathrm{N}_{\mathrm{a}}$ is Avogadro's number and $\mathrm{R}_{\mathrm{a}}$, the gas constant in liter-atmospheres and $\mathrm{N}$ is the number of molecules per $\mathrm{cm}^{-3}$ in a bubble. Therefore, the reaction is substantially complete in less than one and a half seconds.

From the same matrix (eq. 14) we easily evaluate the weighted average photon energy:

$$
\Gamma_{a v}=\frac{\sum_{j=0}^{5} H_{1, j, 0} \cdot H_{1, j, 1}}{\sum_{j=0}^{5} H_{1, j, 0}}=0.548 \mathrm{MeV}
$$

A more detailed look at the bubble reactor for a specific reaction: reduction of carbon dioxide with hydrogen in the presence of a radiation field is shown here. As it turns out for the carbon monoxide generator, the bubble path can be made quite short because the conversion from $\mathrm{CO}_{2}$ to $\mathrm{CO}$ is rapid.

$$
F=3 \pi d \mu \nu
$$

On the other hand, the buoyancy of a bubble in water with diameter $d$ is

$$
F_{b}=\frac{\pi}{6} d^{3} g \rho
$$

Here $\mu$ is the kinematic viscosity for water; $g$ the acceleration of gravity: $980.7 \mathrm{~cm}^{2} / \mathrm{sec} ; \rho$ the liquid density: $1.0 \mathrm{gm} / \mathrm{cm}^{3}$. Equating the two forces yields an expression for the bubble's limiting upward velocity.

The bubble's rising speed is determined by equating the bubble drag to its buoyancy.

$$
v=\frac{1}{18 \mu} g d^{2} \rho
$$

Let the bubble diameter be $0.2 \mathrm{~mm}$, then with the kinematic viscosity for water at 50 degrees $\mathrm{C}$ (cooling pond temperature) as $5.541 \times 10^{-3} \mathrm{~cm}^{2} / \mathrm{sec}$ : the bubble terminal velocity is $3.9 \mathrm{~cm} / \mathrm{sec}$. Assume the bubble rise stabilizes in 10 bubble diameters, then the terminal Reynolds number is a respectable 14.2 denoting laminar-flow. Hence these bubbles will rise in straight columns.

Manufacturing a bubble plate with the required hole diameter and the great number of holes needed is accomplished by laser drilling. Solid state, pulsed lasers have been used for some time now to drill very small diameter holes in materials without a significant kerf because most of the excavated material evaporates in the laser flux. Therefore, manufacturing a plate to meet desired specifications should be relatively easy to accomplish. Here we note that bubble dwell time in the radiation field is a function of the bubble volume that depends in turn on the bubble plate 
hole size. Therefore, hole size determines the dwell-time the reactants spend in the radiation field: the choice optimizes the production rate of a particular product.

The dimensions of the bubble chamber annulus width mentioned above: inner radius $\mathrm{a}=13.5 \mathrm{~cm}$ and annulus radius is $\mathrm{b}=15.6 \mathrm{~cm}$. Therefore, the area is $A=\pi\left[(a+b)^{2}-a^{2}\right]=$ $2.088 \times 10^{3} \mathrm{~cm}^{2}$. Each square centimeter will support $1.111 \times 10^{3} 0.02 \mathrm{~cm}$ diameter holes with a $0.01 \mathrm{~cm}$ spacing between holes (assume contiguous holes with a fictional $0.03 \mathrm{~cm}$ diameter). Hence the whole plate will deliver $2.32 \times 10^{6}$ bubbles at a rate determined by the hole size and the plenum pressure. The estimated detachment time is the time interval a bubble will rise by its diameter according to (19) above. Hence the detachment time is $0.02 / \mathrm{v}=5.13$ milliseconds. The volume of gas per second emitted from the plate is 1.89 liters/sec.

A short list of possible reactions that reduce $\mathrm{CO}_{2}$ in a radiation field

Here is a list of possible reactions created by radiation and a suitable oxygen acceptor. The first of these is the simple reduction of carbon dioxide with hydrogen gas in a radiation field and the subject of our detailed analysis. Note the differences in products obtained by simply changing the mole ratio of hydrogen to carbon dioxide shown in the first three lines.

(a) $\mathrm{CO}_{2}+\mathrm{H}_{2} \rightarrow \mathrm{CO}+\mathrm{H}_{2} \mathrm{O}$

(b) $\mathrm{CO}_{2}+2 \mathrm{H}_{2} \rightarrow \mathrm{H}_{2} \mathrm{CO}+\mathrm{H}_{2} \mathrm{O}$

(c) $\mathrm{CO}_{2}+3 \mathrm{H}_{2} \rightarrow \mathrm{CH}_{3} \mathrm{OH}+\mathrm{H}_{2} \mathrm{O}$

(d) $\mathrm{CO}_{2}+2 \mathrm{H}_{2}+\mathrm{CH}_{4} \rightarrow 2 \mathrm{CH}_{3} \mathrm{OH}$

(e) $\mathrm{CO}_{2}+2 \mathrm{H}_{2}+\mathrm{C}_{2} \mathrm{H}_{6} \rightarrow \mathrm{CH}_{3} \mathrm{OH}+\mathrm{C}_{2} \mathrm{H}_{5} \mathrm{OH}$

(f) $\mathrm{CO}_{2}+2 \mathrm{H}_{2}+\mathrm{C}_{3} \mathrm{H}_{6} \rightarrow \mathrm{C}_{4} \mathrm{H}_{8} \mathrm{O}+\mathrm{H}_{2} \mathrm{O}$ butyraldehyde isomers

$(\mathrm{g}) \mathrm{CO}_{2}+\mathrm{H}_{2}+\mathrm{C}_{4} \mathrm{H}_{8} \mathrm{O}(\mathrm{i}-$ prop $) \rightarrow 0.5\left(\mathrm{CH}_{3}\right)_{2} \mathrm{CO}+\mathrm{CH}_{3} \mathrm{OH}+\mathrm{H}_{2} \mathrm{O}$ acetone

(h) $\mathrm{CO}_{2}+\mathrm{H}_{2}+\mathrm{C}_{2} \mathrm{H}_{4} \rightarrow \mathrm{C}_{3} \mathrm{H}_{4} \mathrm{O}+\mathrm{H}_{2} \mathrm{O}$ Acrolein

(i) $\mathrm{CO}_{2}+$ cyclic chemicals $\rightarrow$ ??

Carbon monoxide, formaldehyde, methanol and ethanol, acrolein, acetone, butyraldehyde isomers, ketones, ketenes plus the reduction of carbon dioxide by cyclic compounds all are endothermic reactions at or near room temperature, and all become exothermic while bombarded by gamma ray generated fast electrons.

The first three reactions show different products are created by simply controlling the mole ratio of hydrogen to carbon dioxide. Clearly reaction (d) is a superior way of producing methanol compared with (c) in addition to the fact that (d) requires another waste gas, methane. Replacing methane with ethane and propane lead to efficient reactions for methanol, ethanol, and butyraldehyde. Hydrogen plus i-propanol and carbon dioxide in a radiation field produces acetone and methane as revealed in reaction $(\mathrm{g})$.

Also note that all products are either completely miscible in water or are quite soluble meaning that the chemical cell water must be processed by either fractional distillation or a newer, less energy-intensive method ${ }^{17}$ to recover the valuable feed stock chemical. 
In the list above, reactions (e), (f), (g) and (i) have slightly exothermic enthalpies, but the entropy term cannot be ignored. Therefore, these reactions are still good candidates for radiation initiation. The question mark following the cyclic chemical hydrogen donors indicates lack of information how the molecules decompose in a radiation field.

Generally, as the bubbles rise, they grow smaller as carbon monoxide and water vapor replaces carbon dioxide and hydrogen. Moreover, if in other cases, the reaction product is soluble, then the reaction is further forced to its product side because of the phase-change. In addition, according to (19) the bubbles will slow down as they grow smaller leading to a froth layer in the reaction vessel as one of the products changes its phase.

Let the reduction of carbon dioxide to the monoxide by

\begin{tabular}{||l|l|l|}
\hline Reactant & $\begin{array}{l}\Delta \mathrm{G} \\
(\mathrm{KJ} / \mathrm{mol})\end{array}$ & $\begin{array}{l}\text { Electron } \\
\text { Fraction, } \\
\alpha\end{array}$ \\
\hline $\mathrm{CO}_{2}$ & -394.9 & $11 / 12$ \\
\hline $\mathrm{H}_{2} \mathrm{O}$ & -228.61 & $5 / 12$ \\
\hline $\mathrm{CO}$ & -137.16 & $7 / 12$ \\
\hline $\mathrm{H}_{2}$ & --- & $1 / 12$ \\
\hline
\end{tabular}

Table 3. Required reaction data molecular hydrogen illustrate the method. To proceed further, we need to estimate the time it takes for the reaction to go to completion, and for that calculation we need the Gibb's Energies of formation for carbon dioxide, carbon monoxide and water, the gas constant, and cooling pond temperature. Table 3 shows the reaction's Gibb's energies. In addition to this information, we also need the normalized atomic numbers for these molecules shown in the last column in the table. The fast electrons generated by Compton scattering in the water will scatter preferentially from the larger electron charge densities of the various reactant molecules.

There are 24 electrons shared by the reactants and products. We expect the denser atomic plasmas to be struck more often than the less dense ones.

$$
\begin{aligned}
& \Delta G=\Delta G_{C O}+\Delta G_{\mathrm{H}_{2} \mathrm{O}}-\Delta G_{\mathrm{CO}_{2}}=28.02 \mathrm{KJ} / \mathrm{mol} \\
& \Delta g=\Delta G_{\mathrm{CO}} \alpha_{C O}+\Delta G_{\mathrm{H}_{2} \mathrm{O}} \alpha_{\mathrm{H}_{2} \mathrm{O}}-\Delta G_{\mathrm{CO}_{2}} \alpha_{\mathrm{CO}_{2}}=186.26 \mathrm{Kj} / \mathrm{mol}
\end{aligned}
$$

Hence according to the first line, the reaction in the absence of radiation is weakly endothermic, and thus does not go spontaneously. However, the second factor makes the overall reaction in the presence radiation very exothermic. According to the analysis presented above, the equilibrium constant is:

$$
K(T, \tau)=K_{0} e^{\frac{(\Delta g f \tau) \cdot 1000}{R_{g} T}}=e^{-\frac{\Delta G 1000}{R_{g} T}} \cdot e^{\frac{(\Delta g f \tau) \cdot 1000}{R_{g} T}} ; \quad \tau_{e}=\frac{\Delta G}{f \Delta g}=2.952 \mathrm{secs}
$$

The second equation is the elapsed time for which the two exponential factors are equal, and the equilibrium constant is one.

It is helpful to visualize the reaction progress as a plot of the products and reactants versus $\xi$ exposure time. The reaction extent $\xi(\xi=1$ reaction complete, $\xi=0$, no reaction products $)$ is a function of the gas dwell-time in the radiation field. It records the transition from endothermic to exothermic states as a function of dwell. The tabular data plotted in Figure 4 was determined by 
numerically solving (13) using the obvious stoichiometry for the reaction (20-a); the definitions of the mole fractions $\chi(\xi)$; and the machinery set up in (12).

The figure reveals that the reaction is essentially complete in three seconds, consistent with the prediction in (eq. 22). Thus, the individual reaction 'donut' chambers need be no thicker than $2.994 \times 2.952=8.84 \sim 9 \mathrm{~cm}$ high.

Each fuel rod cluster will support 43 donut reactors, creating 2.81 liters $/ \mathrm{sec}$ times $43=121$ liters/sec per central assembly. If a typical cooling pond has 1000 assemblies; then 111 of these are available to generate the product--carbon

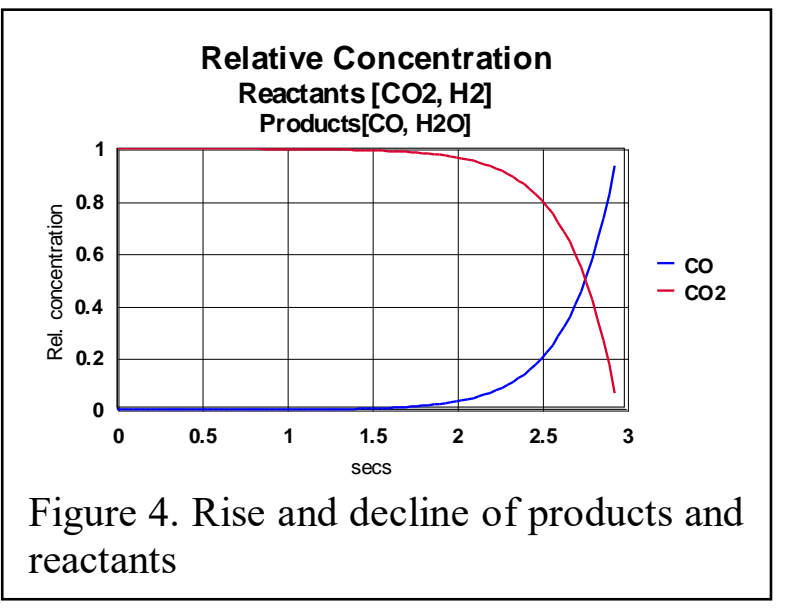
monoxide.

The hundred or so cooling ponds in this country are stuffed to full capacity with spent fuel-rod assemblies. Typically, this means each pond contains about 1000 fuel rod assemblies to be divided into 111 assembly clusters suggested by the geometry in figure 2. Each of these clusters is a separate chemical factory.

In principle, a single well-managed cooling pond could create 121 x $111=13,431$ liters of product gas per second. At a nominal pressure of $1.3 \mathrm{~atm}$, and at a temperature of 330 degrees $\mathrm{K}$, this gas volume corresponds to 659 moles of carbon monoxide produced each second or 2.37 $\mathrm{x} 10^{6}$ moles per hour or 66.4 tonnes per hour yielding a maximum of 0.58 megatonnes/year. If the other possible chemical reactions have approximately the same parameters, we can estimate their productivity by observing that all of them will consume about the same amount of carbon dioxide per unit time, namely 659 moles of carbon dioxide/sec. If all cooling ponds were enlisted in carbon monoxide production, the yearly output would be 58 MT comparable to a contemporary petroleum industry plant's output.

It is true that radiochemical carbon monoxide production by this technique will be small compared to the chemical industry's output and if restricted to present cooling pond number, however its lower cost (no external power needed), environmentally friendly production, and greater purity (no petrochemical contaminants) may establish a secure niche for this process. Moreover, assuming the same parameters but using different hydrogen reductants (eq. 20), it can produce other feedstock chemicals with the same purity and environmental care.

If this concept is tied to existing cooling ponds, then at most, it is a boutique industry with some competitive advantages over petrochemically derived chemical feedstocks. But the pressure of overfilled cooling ponds will drive the Department of Energy to permit transport of large caissons filled with spent assemblies to permanent storage sites. When this occurs, then it will be possible to build dedicated chemical factories on the top of cooling ponds not co-located with nuclear power plants. Scale is no longer limited as these prepared ponds could house tens of thousands of spent assemblies. Now the production capacity is limited only by the marketplace and this new chemical industry could easily disrupt the present petrochemical competition based on a lower cost, purer, high volume product or products. 
References

1. C. Das Neves, et al., "A Diagonal approach to chemical recycling of carbon dioxide: Organocatalytic transformation for the reductive functionalization of $\mathrm{CO}_{2}$," Angew. Chem. Int. Ed., 51, 187-190, 2012.

2. J. L. DiMeglio, J. Rosenthal, "Selective conversion of $\mathrm{CO}_{2}$ to $\mathrm{CO}$ with high efficiency using an inexpensive bismuth-based electrocatalyst," J. Am. Chem. Soc., 135 (24): 87988801, 2013.

3. Q. Lu et al., A Selective and efficient electrocatalyst for carbon dioxide reduction, Nature Commun, $\underline{5}, 3242,2014$.

4. H. A. Duong, et al., "Regioselective copper-catalyzed carboxylation of allylboronates with carbon dioxide," Organic Letters 15, 4034-37, 2013.

5. Katie Lamb, "Carbon Capture and Conversion must not rely on Rare Metals," Phys. Org./news/2019-01-carbon-capture-conversion-rare-metals.html, https://blogs.ei.columbia.edu/2019/29/co2-utilization-profits/

6. Renee Cho, "Capturing Carbon's Potential: These Companies are Turning $\mathrm{CO}_{2}$ into Profits," State of the Planet, Earth Institute, Columbia University, 3/20/2020.

7. S. E. Jensen, E. Nonbøl, "Description of the Magnox type of Gas Cooled Reactor (MAGNOX), Risø National Laboratory, Roskilde, Denmark NKS/RAK-2(97) TR-C5, November 1998.

8. Joon Ha Jin, et al., "Gamma-Radiolysis of Carbon Dioxide (I) Effect of Addition of Alcohols and Aldehydes," Journal of the Korean Chemical Society, 26, No. 3, p135, 1982

9. Joon Ha Jin, et al., "Gamma-Radiolysis of Carbon Dioxide (IV). Effect of the Addition of Alcohols on the Gamma Radiolysis of Gaseous Carbon Dioxide," Bulletin Korean

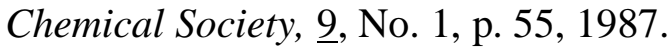

10. Sang Up Choi, et al., "Gamma-Radiolysis of Carbon Dioxide (V). Radiolysis of Carbondioxide Alcohol Mixtures," Journal of the Korean Chemical Society, 35, No. 11991.

11. W. H. Beattie, "The radiolysis of mixtures of carbon dioxide and hydrogen," International Journal of Chemical Kinetics, 4, Issue 4, 463-477, 1972.

12. R. Kummler, C. Leffert, K. Im, R. Piccirelli, L. Kevan, and C. Willis,"A numerical model of carbon dioxide radiolysis," J. Phys. Chem., 1, 2451-2463, 1977.

13. W. M. Garrison, et al, "Reduction of carbon dioxide in aqueous solutions by ionizing radiation," UCRL-1192, 1951.

14. C. Willis, et al., "Carbon monoxide yields in the radiolysis of carbon dioxide at very high dose rates," Canadian Journal of Chemistry, 48, July 1, 1970.

15. Norihiko Fujita, et al, "Radiation-induced $\mathrm{CO}_{2}$ reduction in an aqueous medium suspended with iron powder," Radiation Physics and Chemistry, 44, No. 4, 349-357, Oct. 1994.

16. A. J. Elliot, Monique P. Chenier, and Denis C. Quellette, "g-Value for $\gamma$-irradiated water as a function of temperature," Canadian. J. Chem. $\underline{68}, 712,1990$.

17. Winarto, Daisuke, et al., "Water-methanol separation with carbon nanotubes and electric fields," Nanoscale, $\underline{7}, 12659-12665,2015$. 